\title{
Chandrahigh-resolution Observations of Cid-42, A Candidate Recoiling Supermassive Black Hole
}

\section{Citation}

Civano, F., M. Elvis, G. Lanzuisi, T. Aldcroft, M. Trichas, A. Bongiorno, M. Brusa, et al. 2012. "CHANDRAHIGH-RESOLUTION OBSERVATIONS OF CID-42, A CANDIDATE RECOILING SUPERMASSIVE BLACK HOLE." The Astrophysical Journal 752 (1): 49. https:// doi.org/10.1088/0004-637x/752/1/49.

\section{Permanent link}

http://nrs.harvard.edu/urn-3:HUL.InstRepos:41393173

\section{Terms of Use}

This article was downloaded from Harvard University's DASH repository, and is made available under the terms and conditions applicable to Other Posted Material, as set forth at http:// nrs.harvard.edu/urn-3:HUL.InstRepos:dash.current.terms-of-use\#LAA

\section{Share Your Story}

The Harvard community has made this article openly available. Please share how this access benefits you. Submit a story.

Accessibility 


\title{
CHANDRA HIGH-RESOLUTION OBSERVATIONS OF CID-42, A CANDIDATE RECOILING SUPERMASSIVE BLACK HOLE
}

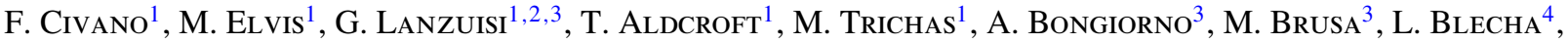

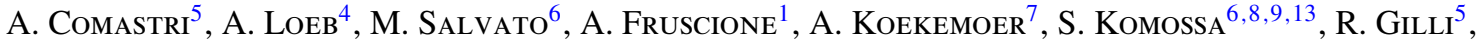 \\ V. Mainieri ${ }^{10}$, E. PiCONCElli $^{2,11}$, AND C. Vignali ${ }^{12}$ \\ ${ }^{1}$ Smithsonian Astrophysical Observatory, 60 Garden Street, Cambridge, MA 02138, USA \\ ${ }^{2}$ INAF-Osservatorio Astronomico di Roma, Via Frascati 33, Monteporzio-Catone 00040, Italy \\ ${ }^{3}$ Max-Planck-Institut für extraterrestrische Physik, Giessenbachstrasse 1, 85748 Garching, Germany \\ ${ }^{4}$ Department of Astronomy, Harvard University, 60 Garden Street, Cambridge, MA 02138, USA \\ 5 INAF-Osservatorio Astronomico di Bologna, Via Ranzani 1, Bologna 40127, Italy \\ ${ }^{6}$ Max-Planck-Institute for Plasma Physics, Excellence Cluster, Boltzmannstrass 2, 85748 Garching, Germany \\ ${ }^{7}$ Space Telescope Science Institute, 3700 San Martin Drive, Baltimore, MD 21218, USA \\ ${ }^{8}$ Fakultaet Fuer Physik, Technische Universitaet Muenchen, James-Franck-Strasse 1/I, 85748 Garching, Germany \\ ${ }^{9}$ Excellence Cluster Universe, Boltzmannstrass 2, 85748 Garching, Germany \\ ${ }^{10}$ ESO, Karl-Schwarzschild-Strasse 2, 85748 Garching, Germany \\ ${ }^{11}$ XMM-Newton Science Operations Centre, ESA, P.O. Box 78, 28691 Villanueva de la Cañada, Spain \\ ${ }^{12}$ Dipartimento di Astronomia, Universitá di Bologna, Via Ranzani 1, Bologna 40127, Italy \\ ${ }^{13}$ Max-Planck-Institut for Radioastronomie, Auf dem Huegel 69, 53121 Bonn, Germany \\ Received 2012 February 15; accepted 2012 April 10; published 2012 May 24
}

\begin{abstract}
We present Chandra High Resolution Camera observations of CID-42, a candidate recoiling supermassive black hole $(\mathrm{SMBH})$ at $z=0.359$ in the COSMOS survey. CID-42 shows two optical compact sources resolved in the $H S T /$ ACS image embedded in the same galaxy structure and a velocity offset of $\sim 1300 \mathrm{~km} \mathrm{~s}^{-1}$ between the $\mathrm{H} \beta$ broad and narrow emission line, as presented by Civano et al. Two scenarios have been proposed to explain the properties of CID-42: a gravitational wave (GW) recoiling SMBH and a double Type 1/Type 2 active galactic nucleus (AGN) system, where one of the two is recoiling because of slingshot effect. In both scenarios, one of the optical nuclei hosts an unobscured AGN, while the other one, either an obscured AGN or a star-forming compact region. The X-ray Chandra data allow us to unambiguously resolve the X-ray emission and unveil the nature of the two optical sources in CID-42. We find that only one of the optical nuclei is responsible for the whole X-ray unobscured emission observed and a $3 \sigma$ upper limit on the flux of the second optical nucleus is measured. The upper limit on the X-ray luminosity plus the analysis of the multiwavelength spectral energy distribution indicate the presence of a star-forming region in the second source rather than an obscured SMBH, thus favoring the GW recoil scenario. However, the presence of a very obscured SMBH cannot be fully ruled out. A new X-ray feature, in a SW direction with respect to the main source, is discovered and discussed.
\end{abstract}

Key words: galaxies: active - galaxies: interactions - galaxies: nuclei

Online-only material: color figures

\section{INTRODUCTION}

Coalescing supermassive black hole (SMBH) pairs should give rise to very strong gravitational wave (GW) events in the universe. After the merging event, the newly formed black hole (BH) can experience a recoil with respect to the center of the galaxy due to asymmetric emission of gravitational radiation (Peres 1962; Bekenstein 1973).

Recent numerical relativity simulations have shown that the recoil velocity can be as high as a $5000 \mathrm{~km} \mathrm{~s}^{-1}$ for rare configurations of $\mathrm{BH}$ spins and mass ratio (Campanelli et al. 2007; Baker et al. 2008; Lousto \& Zlochower 2011a, 2011b). These recoil velocities are higher than the escape velocity of the host galaxy, so the SMBH will be ejected from the system and it may be detected as a displaced quasar (Madau \& Quataert 2004; Loeb 2007). Depending on the amount of material it can carry along, the ejected SMBH will shine for $10^{6}-10^{7} \mathrm{yr}$ (Blecha et al. 2011), until it exhausts the fuel and is not recognizable as a displaced quasar anymore. The recoil may significantly influence the SMBH and galaxy co-evolution (Guedes et al. 2011; Sijacki et al. 2011) and have implications for unified models (Komossa \& Merritt 2008).
In some cases, gas dynamics and stellar interactions may be insufficient to drive the SMBH binary to the GW-dominated regime of inspiral and merger, in which case the binary evolution may stall. The arrival of a third galaxy, with its own SMBH, can induce hardening of the original binary by ejecting the newly arrived SMBH. This mechanism, usually referred to as gravitational slingshot (Saslaw et al. 1974), produces one or more SMBHs displaced from the center of the same galaxy (Hoffman \& Loeb 2007).

While extensive simulations have been performed (Gualandris \& Merritt 2008; Dotti et al. 2010; Blecha et al. 2011), observational searches for recoiling SMBHs are scarce (Bonning et al. 2007; Komossa 2012 for a review). Two candidates have been found by spectroscopic searches for highly shifted broad lines (Komossa et al. 2008; Shields et al. 2009). Another candidate has been discovered using spectropolarimetric observations (Robinson et al. 2010) and a fourth based on optical imaging with Hubble Space Telescope (HST; Batcheldor et al. 2010). Based on X-ray imaging with XMM-Newton, Jonker et al. (2010) found an unusual X-ray source offset from the center of a nearby galaxy and offered three possible explanations, one of which is a recoiling SMBH. 
We (Civano et al. 2010, hereafter C10) studied the properties of CID-42 ( $z=0.359)$ which is the only candidate to date to have both optical imaging and spectroscopic signatures of a recoiling SMBH. In this paper we present the results obtained using the full subarcsecond imaging power of the Chandra High Resolution Camera (HRC-I, 0'.137 pixel $^{-1}$ ) to unambiguously resolve the X-ray emission in CID-42. We assume $H_{0}=$ $70 \mathrm{~km} \mathrm{~s}^{-1} \mathrm{Mpc}^{-1}, \Omega_{M}=0.3$, and $\Omega_{\Lambda}=0.7$.

\section{THE PROPERTIES OF CID-42}

Out of 1761 X-ray detections in the Chandra COSMOS survey (Elvis et al. 2009; F. Civano et al. 2012, submitted), CID-42 is the only source that clearly shows two optical sources (SE and NW) separated by $0.5(2.5 \mathrm{kpc})$ in the $H S T / \mathrm{ACS}$ image and embedded in the same galaxy. In C10, we presented detailed modeling with Galfit (Peng et al. 2002) of the optical surface brightness of CID-42 based on high $(\sim 0)^{\prime} .025$ pixel $^{-1}$ resolution COSMOS HST/ACS (F814W)) image (Koekemoer et al. 2007) to decompose the emission in CID-42. The results showed that the SE optical source has a point-like morphology, typical of a bright active galactic nucleus (AGN), while the NW optical source has a more extended profile with a scale length of $\sim 0.5 \mathrm{kpc}$. In the optical spectra of CID-42 (Very Large Telescope, Magellan), a velocity offset of $\sim 1300 \mathrm{~km} \mathrm{~s}^{-1}$ is measured between the broad and narrow component of the $\mathrm{H} \beta$ line (Figures 5 and 6 of $\mathrm{C} 10$ ).

$\mathrm{C} 10$, linking the offset broad emission line and the point morphology of the SE source, proposed two possible scenarios to explain the properties of CID-42: a GW recoiling $\mathrm{SMBH}$, leaving no $\mathrm{BH}$ in the galaxy center; a Type 1/Type 2 AGN system in the same galaxy where the Type 1 is recoiling due to the slingshot effect in a triple system. Given the point-like morphology and the broad emission lines in the optical spectra, the SE source hosts an active unobscured SMBH in both C10 scenarios. The nature of the NW source, instead, is still not clear. In the GW recoil scenario, the SE SMBH is recoiling from the NW source, which represents the galaxy core from which the $\mathrm{BH}$ has been ejected. In the slingshot scenario, the NW source host an active SMBH, which may be an obscured AGN.

An alternative scenario, reported by Comerford et al. (2009, hereafter Co09), proposes the presence of a dual system in CID42 , where the two merging sources are Type 2 AGNs (from optical emission line diagnostics) spectroscopically resolved in CID-42,

making use of a Keck/DEIMOS spectrum. However, the presence of broad Balmer lines $(\mathrm{H} \beta$ and $\mathrm{H} \alpha$; see the Sloan Digital Sky Survey DR7 quasar catalog in Shen et al. 2011) in the optical spectra of CID-42, does not support the Type 2 (with narrow optical emission lines only) nature proposed by Co09 for at least one of the two sources.

X-ray emission, being insensitive to obscuration up to $N_{\mathrm{H}} \sim$ $10^{24} \mathrm{~cm}^{-2}$, is a strong discriminant and can probe the active nature of the optical sources in CID-42 and reveal the actual physical process taking place. Two X-ray-emitting AGNs are expected in the slingshot scenario in $\mathrm{C} 10$ and the Co09 interpretation, while the SE source would be the only powerful X-ray emitter in the GW recoil scenario.

CID-42 was observed in the C-COSMOS survey with Chandra ACIS for $\sim 166 \mathrm{ks}$, but its position was at a large off-axis angle, where the degraded point-spread function (PSF) did not allow the association of the X-ray emission with one or both the optical sources.

\section{CHANDRA OBSERVATION}

\subsection{Data Reduction}

CID-42 was observed on 2011 January 26 (during Cycle 12, observation ID 12810) with the Chandra HRC-I (Murray et al. 1997) camera for $79.3 \mathrm{ks}$. The source was observed on-axis, just $15^{\prime \prime} .2$ away from the aim point. The data were reprocessed using the Chandra software (CIAO 4.3) tool chandra_repro and the latest calibration files (CALDB 4.4.1). The light curve was examined and no flares of either the source or the background were detected. The HRMA artifact, ${ }^{14}$ a feature identified in the central arcsecond of the Chandra PSF, was located, by using the tool make_psf_asymmetry_region, in the SE direction (Figure 1 red region, top panel), opposite to the optical NW source position.

Though accurate astrometry is already applied to Chandra data, we have run wavedetect to detect sources to be used for the fine relative alignment between the HRC-I and the COSMOS $H S T /$ ACS images. We selected the four sources at the smallest off-axis angles and with enough counts to properly determine their X-ray position. One of the four sources is a galaxy with extended optical morphology which has been discarded from the sample. Three sources were used to align the X-ray to the optical image. A small correction was applied in the $y$ - $\left(00^{\prime} .06\right)$ and $x$-directions $(-0 ! 2)$. The final relative astrometry error is $\sim 0{ }^{\prime} .01$.

\subsection{Data Analysis}

A single bright source is clearly visible in the unbinned data shown in Figure 1 (top panel). The overlaid contours show the HST/ACS (green lines) smoothed image. The position of the SE optical source is co-incident with the peak of the X-ray emission, as also shown in Figure 1 (bottom panel). No obvious $\mathrm{X}$-ray source is present at the optical position of the NW source (marked with a cross in Figure 1).

The number of net counts extracted in a $1^{\prime \prime}$ radius circle is $379 \pm 20$. Given that the spectral resolution of the HRC-I is very limited compared to ACIS and that the spectral shape of CID-42 has not changed in the past (C10), we assumed that the $\mathrm{X}$-ray emission has a soft spectrum as observed in previous observations $\left(\Gamma=2\right.$ and $\left.N_{\mathrm{H}, \mathrm{Gal}}=2.7 \times 10^{20} \mathrm{~cm}^{-2}\right)$ to estimate the total flux of $\sim 9 \times 10^{-14} \mathrm{erg} \mathrm{cm}^{-2} \mathrm{~s}^{-1}\left(L_{\mathrm{X}}=3 \times 10^{43} \mathrm{erg} \mathrm{s}^{-1}\right)$ in the $0.5-10 \mathrm{keV}$ band.

\subsubsection{D Imaging Analysis}

In order to assess the presence of a second X-ray source and determine the flux ratio between the SE and NW optical sources, we performed a two-dimensional (2D) imaging fitting. To model the PSF, we simulated a PSF image at the same off-axis angle of CID-42, by using Chart ${ }^{15}$ and MARX. ${ }^{16}$ All fits were performed in Sherpa (Freeman et al. 2001) using modified Cash statistics and the Nelder and Mead optimization method.

We first measured the FWHM of the PSF by using a 2D Gaussian model, which well represents the PSF shape at the aim point. We then used a three-component model to fit CID-42 X-ray emission: a constant background plus two 2D Gaussian components which FWHM matches the PSF. A family of fits was performed, using a grid of parameter starting points to ensure the proper sampling of the multidimensional fit space. We

\footnotetext{
14 http://cxc.harvard.edu/ciao/caveats/psf_artifact.html

15 http://cxc.harvard.edu/chart/index.html

$16 \mathrm{http}: / /$ space.mit.edu/CXC/MARX/
} 

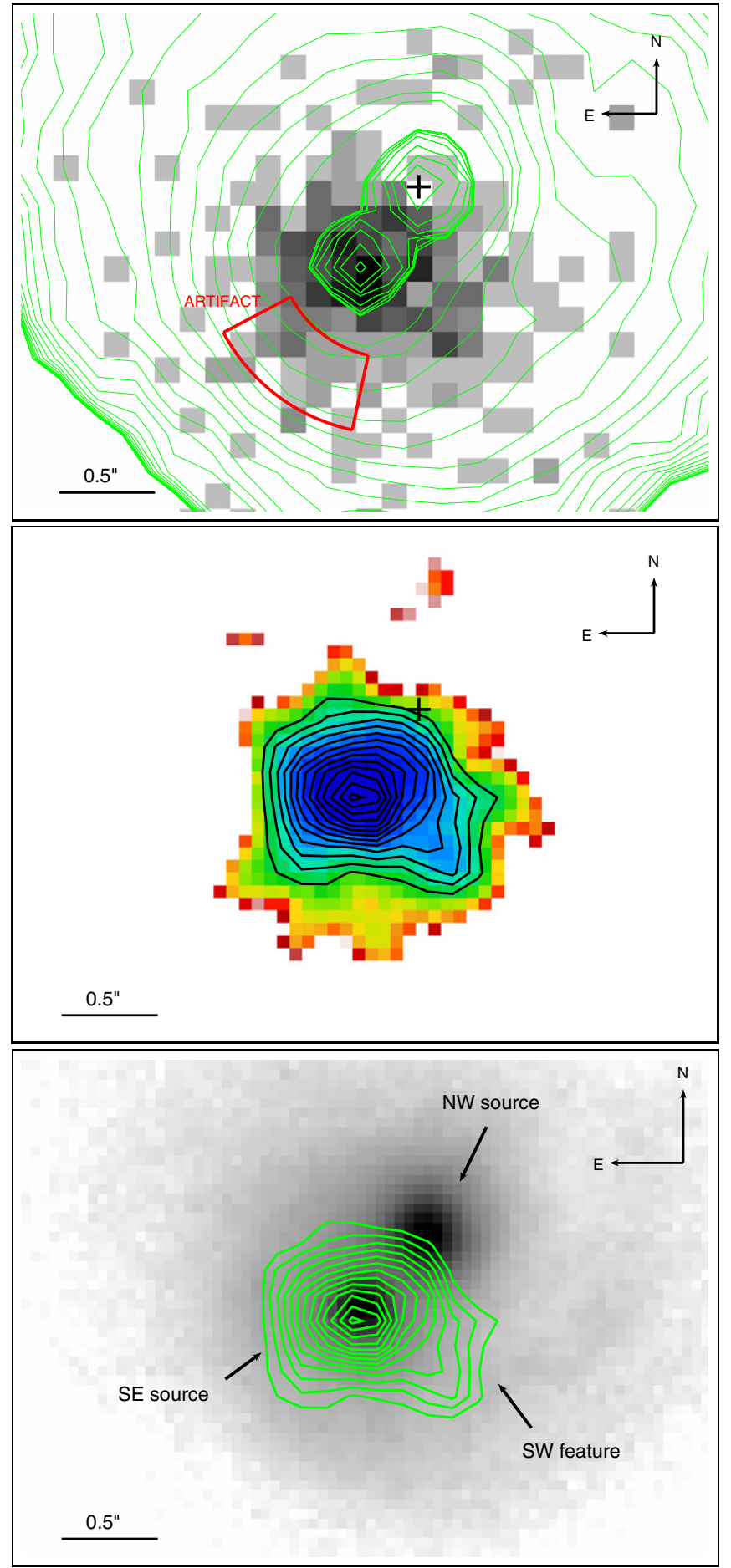

Figure 1. Top: Chandra HRC-I X-ray full-band image with HST/ACS contours overlaid (green). The red solid line represents the HRC artifact region. The cross indicates the position of the NW source determined using the HST/ACS contours. Middle: adaptively smoothed Chandra HRC-I X-ray full-band image with a 3 pixel radius Gaussian kernel. X-ray contours are overlaid (black solid line). Bottom: HST/ACS (filter F814W) image with the X-ray (green) contours overlaid. The NW and SE optical sources have been labeled together with the SW feature. The three images are on the same WCS scale and relative astrometry correction has been applied.

(A color version of this figure is available in the online journal.)

first left the centroid positions of the two Gaussian components free to vary. While the fitted centroid of the main source is consistent (within $<0.2$ pixel in the $x$-direction and $\sim 0.7$ pixel in the $y$-direction) with the optical position of the SE source, the position of a second X-ray source is not constrained. We then fixed the position of the second Gaussian to be within few pixels from the optical position. We measured an upper limit on the ratio between the amplitude of the two Gaussians of $<4.5 \%$ at $3 \sigma$ level (Figure 2), representing the ratio between the intensity of the two sources. By using the amplitude ratio measured with the 2D fitting, we find that the SE optical source is responsible for all of the X-ray emission, while the $3 \sigma$ upper limit on the contribution of the NW optical source is $\sim 4.5 \%$ (17 out of 379 total counts), corresponding to an X-ray total flux of $F_{\mathrm{X}}<3 \times 10^{-15} \mathrm{erg} \mathrm{cm}^{-2} \mathrm{~s}^{-1}$.

\subsubsection{Source Profile Analysis}

Inspection of the X-ray image shows an elongation toward the SW direction (Figure 1, middle panel). To quickly assess the significance of the asymmetry, we extracted the counts using the quadrant method (Elvis et al. 1983). We divided the region into a set of eight inner and outer quadrants with a radius of 0 '.3 and $1^{\prime \prime}$, respectively. We moved the center around the position of the SE source slightly, until we found the location such that the most equal number of counts in each of the inner quadrants (n1 to n4) is measured. In Figure 3 the net counts in each quadrant are reported. In two of the outer regions (n5 and n6) we have a similar number of counts as expected from the symmetry of the PSF. The $n 7$ quadrant is affected by the artifact region. Instead, there is a $5 \sigma$ excess of $\sim 32.5$ counts in the region $n 8$ with respect to region $\mathrm{n} 5$ and $\mathrm{n} 6$.

A more detailed fitting procedure was then employed. We extracted the counts using regions of 0 '. $16 \times 2$ '.5 (plotted in the panel enclosed in Figure 4, left), to build the X-ray source profile (blue circles in Figure 4, left panel) in a direction perpendicular to the line joining the two optical sources. The profile shows a significant deviation from the PSF profile, extracted at the same position in the same direction (black dashed line in Figure 4, left). By fitting the source profile with two PSF profiles we find that the amplitude of the SW X-ray feature contains $\sim 18 \%$ of the amplitude of the main source.

We also extracted another source profile along the line joining the optical sources (regions in the panel enclosed in Figure 4, right). The source profile has the same shape of the PSF profile (in the same direction) but the intensity is higher than in the perpendicular direction. When fitting the profile, a component to fit the SW X-ray feature is required with a peak intensity of $\sim 18 \%$ of the main source, in agreement with the profile extracted in the perpendicular direction. The SW feature is thus responsible for the larger number of counts at the peak of the radial profile along this direction. Instead, we can fit a component at the NW optical source, with intensity consistent with the finding of the $2 \mathrm{D}$ fitting ( $<4 \%$ of the main source), only by freezing its position.

The SW X-ray feature source is offset $\sim 0.5(\sim 2.5 \mathrm{kpc}$ projected) from the center of the main point-like source. We performed extensive simulations (2000 runs) with MARX to determine whether the SW feature could be due to an irregular shape of the PSF. The probability that only a single point source is present is $<0.005$.

\section{DISCUSSION}

The spatial resolution of HRC-I allows us to confirm the presence in CID-42 of only one X-ray emitter and that the X-ray emission is centered on the SE source. A $3 \sigma$ upper limit is measured at the position of the NW optical source. In agreement with both recoil scenarios ( $\mathrm{GW}$ and slingshot), the SE 


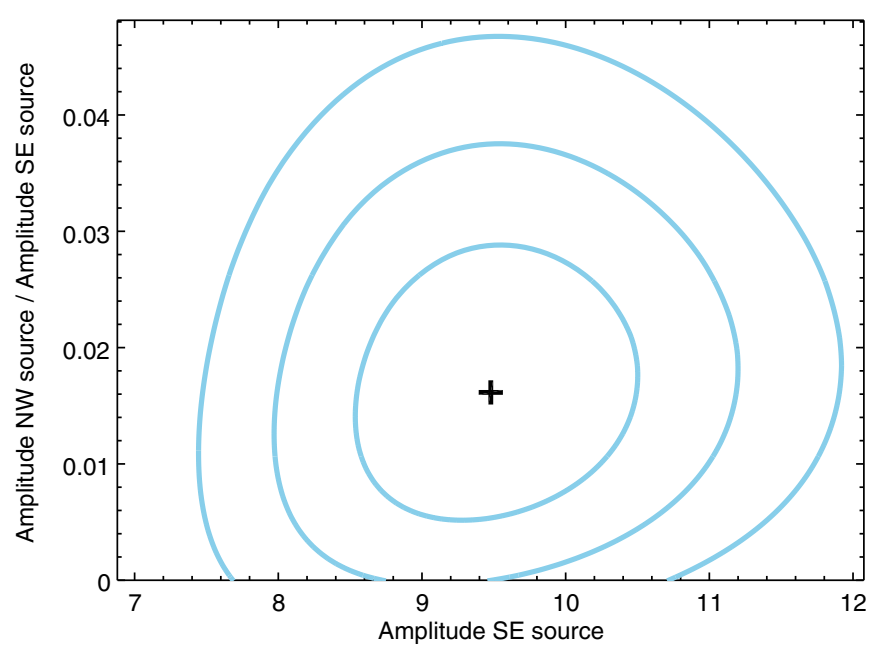

Figure 2. Confidence contours of fit statistic between the ratio between the amplitude of the two components and the amplitude of the brightest component. The three contours are drawn at $1 \sigma, 2 \sigma$, and $3 \sigma$ confidence levels.

(A color version of this figure is available in the online journal.)

optical source has an X-ray emission consistent with being an unobscured active $\mathrm{SMBH}$, as measured in the X-ray spectra in $\mathrm{C} 10$, and in agreement with the broad emission lines in the optical spectra (C10). Obscuration from a torus cannot be present in the SE source in the recoil scenario of $\mathrm{C} 10$, because, when the $\mathrm{SMBH}$ recoils, only the most tightly bound regions (disk and broad line regions) are carried with it, and the more distant regions (torus and narrow line regions) are left behind (Merritt et al. 2006; Loeb 2007; Komossa \& Merritt 2008). The broad emission lines and the unobscured X-ray spectrum of the SE source do not match well with the finding of Co09, who reported two narrow line (Type 2) AGNs.

The HRC flux measured for the SE optical source is consistent, within the errors, with the flux observed in the nine XMM-Newton and Chandra observations, taken over five years (C10; Figure 2, right). The upper limit estimated for the NW source is $F_{\mathrm{X}}<3 \times 10^{-15} \mathrm{erg} \mathrm{cm}^{-2} \mathrm{~s}^{-1}$, at least $\sim 50$ and $\sim 15$ times below the maximum $\left(F_{\mathrm{X}}=1.5 \times 10^{-13} \mathrm{erg} \mathrm{cm}^{-2} \mathrm{~s}^{-1}\right.$ in $2005)$ and the minimum $\left(F_{X}=5 \times 10^{-14} \mathrm{erg} \mathrm{cm}^{-2} \mathrm{~s}^{-1}\right.$ in 2007) observed for CID-42. The upper limit on the X-ray luminosity of the NW optical source is $L_{\mathrm{X}}<10^{42} \mathrm{erg} \mathrm{s}^{-1}$, which can be consistent either with emission from a normal or star-forming galaxy, or with a small fraction of scattered emission from a highly obscured AGN, or with a quiescent SMBH.

Star formation rate (SFR) in the system could provide information on the nature of the X-ray emitter. We used the spectral energy distribution (SED), as published in C10, to compute the SFR. We normalized a standard Elvis et al. (1994) quasar template to the SE optical source magnitude measured in $\mathrm{C} 10$ and subtracted its contribution from the SED at all wavelength. We then performed a multi-component fitting using the $\chi^{2}$ minimization technique and several observed galaxies, quasar, and hybrids (galaxy plus quasar) templates following the method of Ruiz et al. (2010) and Trichas et al. (2012). In the SED fitting we also included the SPIRE far-infrared data points from the Herschel Multi-tiered Extragalactic Survey (HerMES; Oliver et al. 2012) not available to C10. The best fit $\left(\chi^{2} /\right.$ dof $\left.=1.1\right)$ is produced by a young star-forming galaxy template (NGC 7714; Brandl et al. 2004; Ruiz et al. 2010) with a luminosity of $L_{8-1000 \mu \mathrm{m}}=6 \times 10^{44} \mathrm{erg} \mathrm{s}^{-1}, \mathrm{SFR}=25 M_{\odot} \mathrm{yr}^{-1}$, computed using the Kennicutt (1998) relation, and age of the burst of 3-5 Myr. The statistical significance of the best fit is

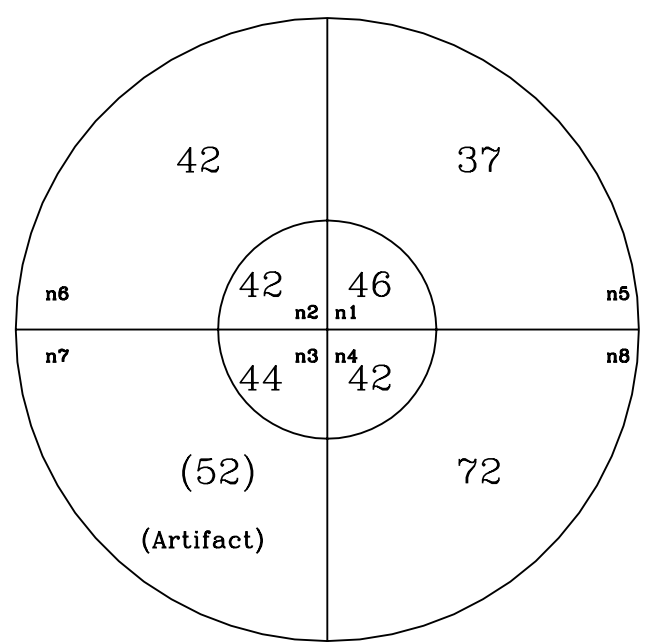

Figure 3. Number of counts in each quadrant. The center corresponds approximately to the SE source. Inner circle radius $=00^{\prime \prime} 3$, outer circle radius $=1^{\prime \prime}$.

$9 \sigma$ above the second possible solution. The SFR derived and the infrared luminosity are consistent with the upper limit on the X-ray luminosity according to the relation between the three quantities derived for local galaxies (Ranalli et al. 2003). This fitting thus favors the presence of a young star-forming galaxy in CID-42 in agreement with the measure of the X-ray upper limit for the NW optical source. However, the SFR rate measured could be produced not only by a young stellar cluster in the NW source, but could be the sum of the overall star formation in the system.

Since the total X-ray emission is dominated by the SE optical source, we cannot probe directly the presence of X-ray obscuration in the NW source from the X-ray spectral analysis. An indirect probe of strong obscuration could be the presence of a strong (with an equivalent width of $1-2 \mathrm{keV}$ ) iron emission line (Matt et al. 2003). We indeed detected the FeK $\alpha$ line in CID-42 spectra (C10) with a rest-frame equivalent width of $\mathrm{EW}=142_{-86}^{+143} \mathrm{eV}$ in the XMM-COSMOS spectrum (where the continuum was higher) and $\mathrm{EW}=\sim 550 \pm 260 \mathrm{eV}$ in the Chandra spectrum (where the continuum was lower), consistent with the EW typical of Seyfert 1 objects (Yaqoob \& Padmanabhan 2004). If we assume that the iron lines observed were entirely produced by the NW optical source, which continuum emission is 25 times fainter than the observed one, thus its EW should be a factor 25 higher, $\sim 25 \mathrm{keV}$ or more, an unphysical value.

A quiescent SMBH could still be hosted by the NW optical source. However, observations in the local universe have shown that in gas-rich and or disturbed systems (e.g., MW, NGC 1068, NGC 4151, Mrk 348), but also in early-type galaxies (Soria et al. 2006; Pellegrini 2010), active SMBHs are ubiquitous even if their activity could be sometime very low.

\subsection{The SW X-Ray Feature}

The SW X-ray feature has a flux of $\sim \times 10^{-14} \mathrm{erg} \mathrm{cm}^{-2} \mathrm{~s}^{-1}$ $\left(L_{\mathrm{X}} \sim 3 \times 10^{42} \mathrm{erg} \mathrm{s}^{-1}\right)$ in the full HRC band. The probability that this feature is a background source, at $z>z_{\text {CID-42, within }}$ a $1^{\prime \prime} .5$ radius circle from the center of CID-42 is $8 \times 10^{-5}$ (computed using the Gilli et al. 2007 number count predictions).

From the X-ray and optical images and contours (Figure 1), a possible association of the SW X-ray feature and the minor SW optical tail in CID-42 is possible, although this could just be due to a projection effect. 

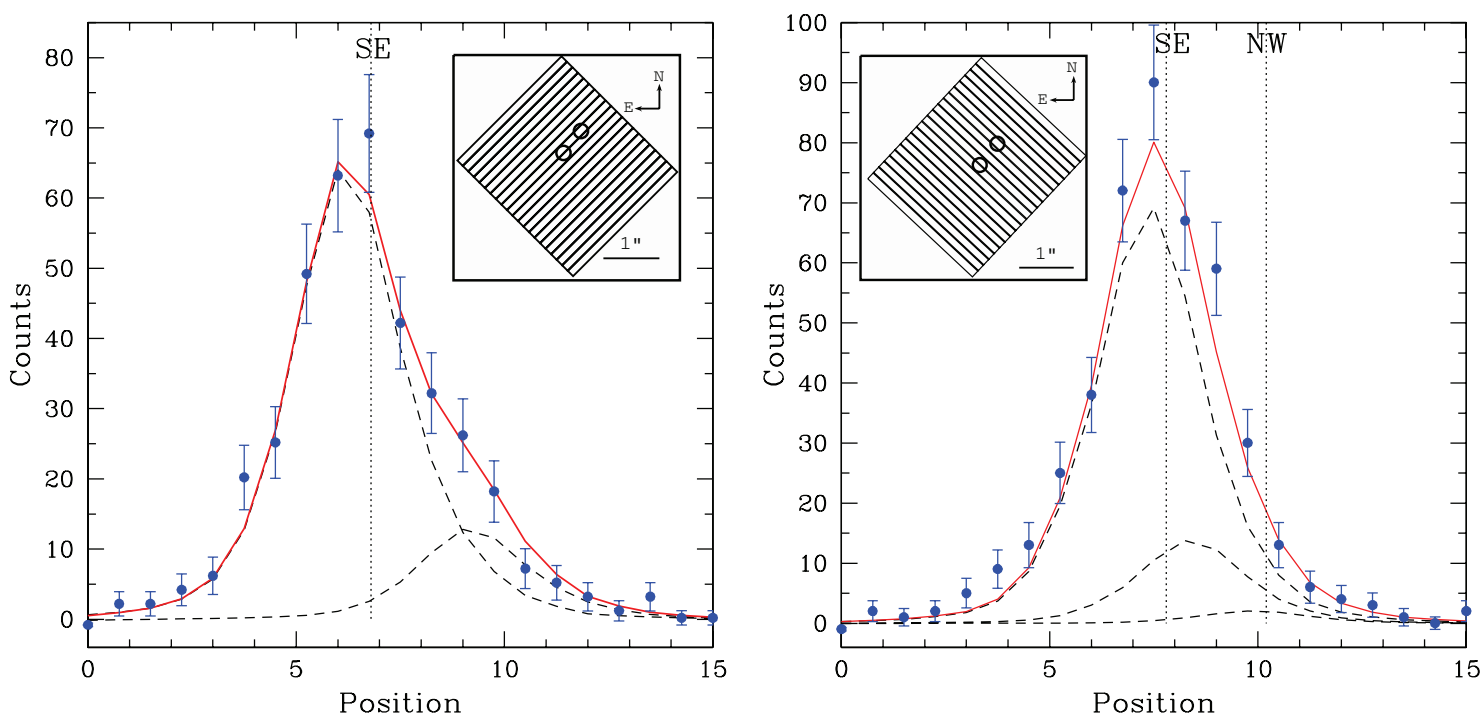

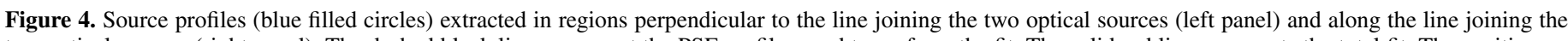

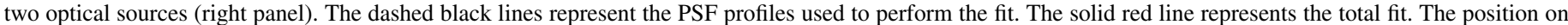

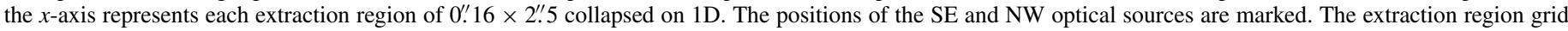
(with the position of the NW and SE sources) is plotted in the sub-panel.

(A color version of this figure is available in the online journal.)

Different physical scenarios can be used to explain the X-ray emission of the SW feature, each consistent with the recoil scenario: (1) Star formation could be the cause of the X-ray emission in the SW feature, with the same justification for the NW source (or, starburst-driven superwind activity). (2) The narrow [O III] emission lines observed in the optical spectra of CID-42, at the same redshift of the host galaxy (see C10), could be produced by photoionization of the galaxy interstellar medium (ISM) by the ejected SMBH, on its way out from the center. This would imply that the SMBH is close to a region of the galaxy disk with high ISM density or molecular clouds, to produce sufficiently high ionization. The same photoionized gas would also produce $\mathrm{X}$-ray emission lines in the soft band $(\lesssim 1 \mathrm{keV})$, as already detected in some Seyfert galaxies (e.g., NGC 4151; Wang et al. 2011). (3) A wind from the accretion disk (Elvis 2000) can produce bicones of gas moving at $\sim 500 \mathrm{~km} \mathrm{~s}^{-1}$ out from the nucleus. Internal shocks may heat enough of this gas to X-ray temperatures to be detectable. The luminosity would depend on the wind kinetic power. (4) The low $L / L_{\text {Edd }} \sim 0.04$ estimated for the SMBH in CID-42 (C10) favors the presence of a jet, as in Galactic BH binaries where jets turn on at or below this Eddington ratio (Done et al. 2007). Though CID-42 is detected at $20 \mathrm{~cm}\left(f_{20 \mathrm{~cm}}=138 \pm 38 \mu \mathrm{Jy}\right.$; VLA-COSMOS survey; Schinnerer et al. 2010), the low resolution (HPBW = 1 1.5) does not allow to associate the radio emission with the whole galaxy, the two optical sources or with the SW feature. CID-42 is radio quiet $\left(R_{L}=\log \left(f_{5 \mathrm{GHz}} / f_{B}\right)=-0.55\right.$; C10), but even radio-quiet AGNs can have weak jets (Ulvestad \& Wilson 1984). Either a jet seen near our line of sight $\left(\lesssim 10^{\circ}\right)$ as in, e.g., 3C273 (Marshall et al. 2001), or a jet at a large angle (e.g., M87; Harris et al. 2009), of which we may be seeing only the brightest component, could produce the SW feature.

\section{FUTURE OBSERVING STRATEGY}

The new HRC X-ray data bring us to a more clear understanding of the nature of CID-42 SE optical source. Even if we now strongly prefer a star-forming nature for the NW optical source, the possibility of a truly Compton-thick AGN still remains open.
The origin of the newly discovered SW X-ray feature is still unclear. In the following we briefly discuss observations that might shed even more light on CID-42.

The major problem in reaching an unambiguous conclusion on the nature of the optical emission line components at different velocities (i.e., broad emission lines and narrow emission lines) is the uncertainty on their spatial location. This is technically challenging for seeing-limited observations because of the small separation (0'.5) between the two optical sources, thus either HST high spatial resolution spectroscopy or integral field spectroscopy with adaptive optics would be the next step. As, for example, we expect to see continuum blue emission and narrow emission lines in the NW source if a cusp of young stars due to a starburst in the central gas reservoir is responsible for the upper limit measured in the X-ray band.

High-resolution multi-frequency radio imaging is essential to locate and clarify the nature of the detected $20 \mathrm{~cm}$ radio emission in CID-42. Jansky-VLA observations in two different bands could provide not only high angular resolution $(<0$ ' 4$)$ to test for a jet in the SW X-ray feature, but also to separate the contribution of the nuclear and the stellar emission to the total radio flux. Moreover, Very Long Baseline Array observations at $20 \mathrm{~cm}$ (PI: E. Middelberg; final resolution of $15 \times 15 \mathrm{mas}^{2}$ ) are ongoing for all the VLA-COSMOS sources including CID-42.

New on-axis X-ray data with Chandra ACIS-S to get both the spectral and spatial resolution (with the help of HRC data) could characterize the nature of the SW feature. Hard X-ray emission is expected in the case of a jet, while soft band emission, due to the presence of unresolved emission lines, is expected if the $\mathrm{SW}$ feature is produced by photoionized gas.

If a very obscured SMBH is hidden in the NW source, this could be detected by NuSTAR (Harrison et al. 2010), which, in its 2 year prime mission, will devote 3.1 Ms to mapping the C-COSMOS area in the 8-60 keV band.

\section{SUMMARY}

We analyzed the high spatial resolution HRC Chandra data obtained for CID-42, using both 2D spatial fitting and 1D radial 
profile fitting. Only one X-ray source is detected. The main $\mathrm{X}$-ray emission is clearly produced by the SE optical source and the flux measured is consistent with previous observations.

A $3 \sigma$ upper limit on the X-ray emission of the NW optical source has been computed and its luminosity, together with the analysis of the multiwavelength SED, favor the presence of a star-forming cluster in the NW source, instead of that of an obscured SMBH. The unambiguous association of the X-ray emission with the SE optical source is consistent with the recoil scenarios proposed by $\mathrm{C} 10$ where only one active $\mathrm{SMBH}$ is expected. We note, however, that the presence of a very obscured active SMBH, as proposed by Co09, cannot be fully ruled out.

Making use of all the observational properties measured for CID-42 (morphology, BH mass, galaxy mass, luminosity, SFR, accretion rate), L. Blecha et al. (2012, submitted) will present a detailed modeling of CID-42 as both a GW recoiling SMBH and a pre-merger, kpc-scale dual AGN.

The discovery of a new X-ray feature, in a SW direction with respect to the main source, makes CID-42 even more intriguing. This feature could be connected with the star formation in the system, or emission from gas ionized by the SMBH or a jet.

Future observations at the optical, $\mathrm{X}$-ray, and radio wavelengths will be proposed to further investigate the peculiar properties of CID-42.

F.C. thanks A. Marinucci, A. Goulding, V. Kashyap, and E. Schinnerer for useful discussions on the X-ray data. The authors thank the referee for the useful suggestions which improved the quality of the manuscript. This work was supported by the NASA Chandra grant GO7-8136A, the Blancheflor Boncompagni Ludovisi foundation, and the Smithsonian Scholarly Studies.

\section{REFERENCES}

Baker, J. G., Boggs, W. D., Centrella, J., et al. 2008, ApJ, 682, L29 Batcheldor, D., Robinson, A., Axon, D. J., Perlman, E. S., \& Merritt, D. 2010, ApJ, 717, L6

Bekenstein, J. D. 1973, ApJ, 183, 657

Blecha, L., Cox, T. J., Loeb, A., \& Hernquist, L. 2011, MNRAS, 412, 2154

Bonning, E. W., Shields, G. A., \& Salviander, S. 2007, ApJ, 666, L13

Brandl, B. R., Devost, D., Higdon, S. J. U., et al. 2004, ApJS, 154, 188

Campanelli, M., Lousto, C. O., Zlochower, Y., \& Merritt, D. 2007, Phys. Rev. Lett., 98, 231102
Civano, F., Elvis, M., Lanzuisi, G., et al. 2010, ApJ, 717, 209

Comerford, J. M., Griffith, R. L., Gerke, B. F., et al. 2009, ApJ, 702, L82

Done, C., Gierliński, M., \& Kubota, A. 2007, A\&AR, 15, 1

Dotti, M., Volonteri, M., Perego, A., et al. 2010, MNRAS, 402, 682

Elvis, M. 2000, ApJ, 545, 63

Elvis, M., Briel, U. G., \& Henry, J. P. 1983, ApJ, 268, 105

Elvis, M., Civano, F., Vignali, C., et al. 2009, ApJS, 184, 158

Elvis, M., Wilkes, B. J., McDowell, J. C., et al. 1994, ApJS, 95, 1

Freeman, P., Doe, S., \& Siemiginowska, A. 2001, Proc. SPIE, 4477, 76

Gilli, R., Comastri, A., \& Hasinger, G. 2007, A\&A, 463, 79

Gualandris, A., \& Merritt, D. 2008, ApJ, 678, 780

Guedes, J., Madau, P., Mayer, L., \& Callegari, S. 2011, ApJ, 729, 125

Harris, D. E., Cheung, C. C., Stawarz, Ł., Biretta, J. A., \& Perlman, E. S. 2009, ApJ, 699, 305

Harrison, F. A., Boggs, S., Christensen, F., et al. 2010, Proc. SPIE, 7732, 77320 S

Hoffman, L., \& Loeb, A. 2007, MNRAS, 377, 957

Jonker, P. G., Torres, M. A. P., Fabian, A. C., et al. 2010, MNRAS, 407, 645

Kennicutt, R. C., Jr. 1998, ApJ, 498, 541

Koekemoer, A. M., Aussel, H., Calzetti, D., et al. 2007, ApJS, 172, 196

Komossa, S. 2012, Adv. Astron., 2012, 364973

Komossa, S., \& Merritt, D. 2008, ApJ, 689, L89

Komossa, S., Zhou, H., \& Lu, H. 2008, ApJ, 678, L81

Loeb, A. 2007, Phys. Rev. Lett., 99, 041103

Lousto, C. O., \& Zlochower, Y. 2011a, Phys. Rev. Lett., 107, 231102

Lousto, C. O., \& Zlochower, Y. 2011b, Phys. Rev. D, 83, 024003

Madau, P., \& Quataert, E. 2004, ApJ, 606, L17

Marshall, H. L., Harris, D. E., Grimes, J. P., et al. 2001, ApJ, 549, L167

Matt, G., Guainazzi, M., \& Maiolino, R. 2003, MNRAS, 342, 422

Merritt, D., Storchi-Bergmann, T., Robinson, A., et al. 2006, MNRAS, 367, 1746

Murray, S. S., Chappell, J. H., Kenter, A. T., et al. 1997, Proc. SPIE, 3114, 11

Oliver, S. J., Brock, J., Altien, B., et al. 2012, MNRAS, in press (arXiv:1203.2562)

Pellegrini, S. 2010, ApJ, 717, 640

Peng, C. Y., Ho, L. C., Impey, C. D., \& Rix, H.-W. 2002, AJ, 124, 266

Peres, A. 1962, Phys. Rev., 128, 2471

Ranalli, P., Comastri, A., \& Setti, G. 2003, A\&A, 399, 39

Robinson, A., Young, S., Axon, D. J., Kharb, P., \& Smith, J. E. 2010, ApJ, 717, L122

Ruiz, A., Miniutti, G., Panessa, F., \& Carrera, F. J. 2010, A\&A, 515, A99

Saslaw, W. C., Valtonen, M. J., \& Aarseth, S. J. 1974, ApJ, 190, 253

Schinnerer, E., Sargent, M. T., Bondi, M., et al. 2010, ApJS, 188, 384

Shen, Y., Richards, G. T., Strauss, M. A., et al. 2011, ApJS, 194, 45

Shields, G. A., Bonning, E. W., \& Salviander, S. 2009, ApJ, 696, 1367

Sijacki, D., Springel, V., \& Haehnelt, M. G. 2011, MNRAS, 414, 3656

Soria, R., Fabbiano, G., Graham, A. W., et al. 2006, ApJ, 640, 126

Trichas, M., Green, P. J., Silverman, J. D., et al. 2012, arXiv:1204.5148

Ulvestad, J. S., \& Wilson, A. S. 1984, ApJ, 285, 439

Wang, J., Fabbiano, G., Elvis, M., et al. 2011, ApJ, 742, 23

Yaqoob, T., \& Padmanabhan, U. 2004, ApJ, 604, 63 